\title{
A comparison of information-searching activities before and after hypothesis formation
}

\author{
TOSHIYA TANAKA ${ }^{1}$ \\ Nagoya City College of Child Education, Owariasahi, Aichi 488
}

\begin{abstract}
Quantitative and qualitative differences in perceptual information-searching activities before and after hypothesis formation were investigated. The task performed was a simple target searching task, in which the location of the target was varied to form a pattern. As indices of information-searching activities, head movements with a restricted visual field were recorded. The following became clear: Before a hypothesis was established, the main strategy was to search for the target after the stimulus was presented. Once the pattern hypothesis was established, however, an anticipative strategy prevailed.
\end{abstract}

Key words: hypotheses, perceptual activities, information-processing, eye movements, head movements, anticipative strategies.

Bruner and Postman closely investigated the relationship between hypothesis and information processing (Bruner \& Postman, 1949; Bruner, 1951; Postman, 1951). According to Gregory's categorization (Gregory, 1974), their studies belong to the "perception as hypothesis testing" paradigm. Other studies conducted by the researchers who are interested in the relationship between hypothesis and perception (Salthouse \& Danziger, 1978; Power, 1978) also regard perception as hypothesis testing.

However, their findings are not always sufficient in that their dependent variables are indirect indices of information-searching activities, such as reaction time (RT) or correct percentages. Direct measurement of eye movements may be more

1 The author would like to acknowledge the continuing guidance and encouragement of Dr. Masami Kajita, Nagoya University. At the same time, many helpful discussions with Dr. Hideo Kojima and Dr. Ryohji Osaka, Nagoya University (Dr. Osaka is now in Aichigakuin University), are gratefully acknowledged. Thanks are also extended to Dr. Alan Fogel, Purdue University and visiting scholar (1983-1984), Nagoya University, and Professor Adelbert George Smith, the Language Center of Nagoya University, for their helpful comments in editing this article. useful for assessing information-searching, but this type of research is laborious at the stage of data analysis. Thus, head movements will be substituted for eye movements.

The purpose of this study is to investigate the formation of a human pattern hypothesis behavior through a simple task. Main dependent variables are RT and head movements. The following experimental hypotheses are to be tested as a pilot study.

1. Before a hypothesis concerning the pattern of the target is established, subjects may scan the problem space freely, so that the RT may be considerably long.

2. After a pattern hypothesis is established, due to the expectation of spatial location, subjects may adopt a strategy in which they fixate the location of the target in advance of the presentation of the target, as indicated by a shorter RT.

\section{Method}

\section{Subjects}

Subjects were eleven undergraduate students. In the course of the experiment, however, two of them were detected as being unable sufficiently to understand instructions. Another two were elimi- 
nated at one stage of analysis because their variance of RT was markedly greater than those of the other subjects. Then only the data for seven subjects (four females and three males) were analyzed.

\section{Materials}

Original stimuli were written on each corner of a rectangle, $53 \mathrm{~mm}$ long and $70 \mathrm{~mm}$ wide. The stimuli were four numerals $(3,6,8,9), 5 \mathrm{~mm}$ wide and $6 \mathrm{~mm}$ high.

The original stimuli containing four numerals were photographed using a $35 \mathrm{~mm}$ monochromatic slide with the aid of Panacopy (KV-3000: National). The location of each numeral was counterbalanced.

\section{Apparatus}

Total display. The apparatus used consisted of four sections, i.e., exposition of stimuli, detection of responses, detection of head movements, and recording of responses and head movements. Figure 1 shows the total arrangement. Details are as follows.

Units of apparatus. 1. Stimulus exposition section. Slides were placed in a slide projector (Perfect: Cabin) and projected on a screen which was set at a distance of $800 \mathrm{~mm}$ from the subject. The screen was a rectangle $800 \mathrm{~mm}$ wide and $550 \mathrm{~mm}$ high, which had four translucent acrylic boards at each corner. Each board was also a rectangle, $110 \mathrm{~mm}$ long and $230 \mathrm{~mm}$ wide. One of the four numerals was exposed on each of these boards.

2. Response detection section. A key box was used, consisting of four soft touch keys mounted on a metal rectangular surface, $72 \mathrm{~mm}$ long and $130 \mathrm{~mm}$ wide. Each key had two microswitches that could function independently. One microswitch of each key was used to report responses. Another one was for the promotion of slides. When a subject pushed one of the keys, the information was sent to the recording section, and at the same time the next slide was projected. Besides the key box, a key for reporting hypothesis formation was used.

3. Head movement detection section. Rays emitted from a $6 \mathrm{~W}$ lamp were reflected from a dentist's mirror worn back-to-front and up-side-down on a subject's head. The rays were received by four CdS-cadmium sulfide-photocells. The photocells were mounted at the bottom of four $40 \mathrm{~mm}$ high black tubes that were set on a wire-mesh net. The

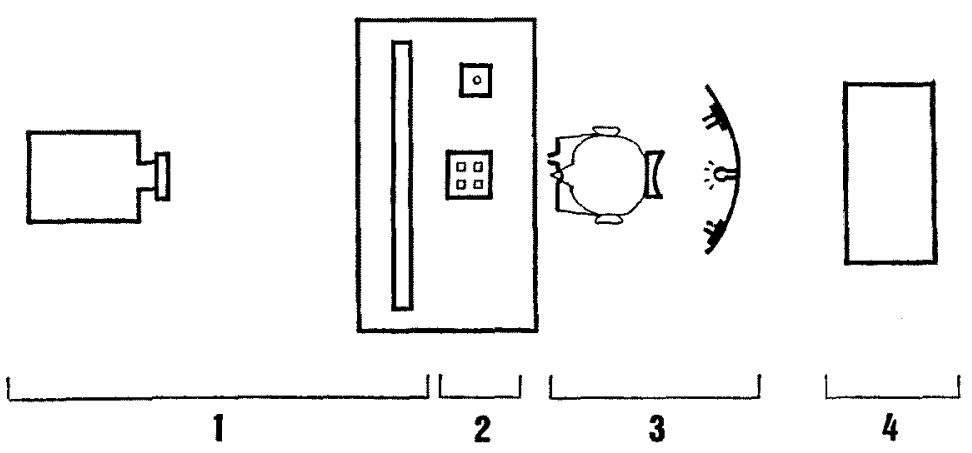

Fig. 1. Arrangement of apparatus.

1) Stimulus exposition section.

2) Response detection section.

3) Head movement detection section.

4) Response and head movement recording section. 
electric current from the CdS photocells was amplified and was conveyed to the micro-computer.

Subjects wore glasses for the purpose of limiting their sight. The left side of the glasses was covered with an opaque plastic board, and the right side was composed of a small frustum of a cone. Subjects could see the screen through a small hole of the frustum. The visual field on the screen was a circle with a diameter of $110 \mathrm{~mm}$ (visual angle was about $8^{\circ}$ ); accordingly subjects had to move their heads to see the stimuli on the screen.

4. Response and head movement recording section. Information about responses, hypothesis formation and head movements were fed through an interface into micro-computer (PC-8001: NEC). An I/O unit (PC-8012: NEC) was used for the purpose of obtaining the real duration time of key-key intervals. The accuracy of the clock was $\pm 5 \mathrm{~ms}$ and the minimum interval it could detect was $40 \mathrm{~ms}$. So it was decided that interval of less than $40 \mathrm{~ms}$ should be regarded as $0 \mathrm{~s}$, and intervals greater than or equal to $40 \mathrm{~ms}$ were recorded to the nearest $1 / 100$ of a second.

\section{Instruction and Task}

Instruction. The task to be performed was a simple target searching task. Subjects were individually instructed that when they found the target they were to press the response key as quickly as possible. After they pushed the response key, the next slide was projected. They were also told that when they found the pattern of the location of the target they were to push the "pattern hypothesis" key only once.

Task. In the practice session, the target was " 6 ", i.e., the task was to find " 6 " and to push the key corresponding to the location of " 6 " on the screen. After the instruction, calibration of instruments was carried out. Five trials were performed in this session.

In the experimental session, the target was " 8 ". It was designed so that " 8 " was presented successively at clockwise varying position; e.g., it was presented on the upper left side of the screen on the first trial, on the upper right side on the second trial and so forth. The number of trials in this session was thirty-six.

\section{Definitions}

Head fixation point (HFP). The location of the CdS photocell which received rays when a subject was fixating a certain numeral on the screen.

Duration of head fixation (HFD). Dura-

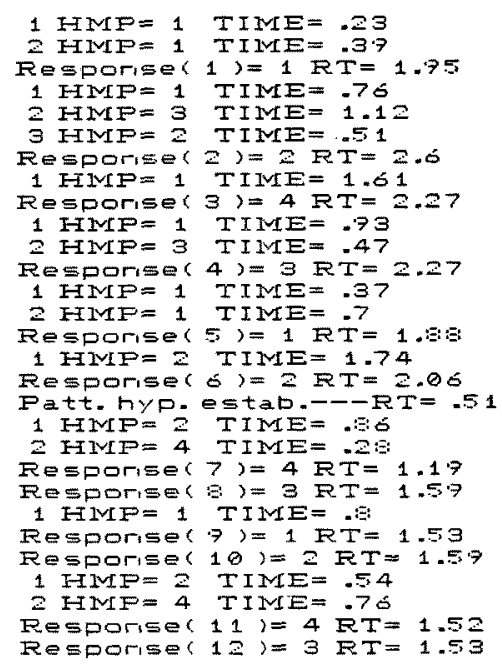

Fig. 2. An example of sequential data.

Left side of the " HMP = " and the numerals in ( ) denote the order of head movements and responses. Right side of them denote the location of head movements and responses as follows;

1: upper left side, 2: upper right side,

3: lower left side, 4: lower right side.

Unit of "TIME" and "RT" is second, and HMP in this figure is equivalent to HFP in the text.

"TIME" and "RT" in this figure are arrayed with two dimensions in the text. $\mathrm{TI}_{i, j}$ denotes time interval of $j$-th head movement in $i$-th response trial. $\mathrm{TI}_{i, 0}$ is equal to " $\mathrm{RT}$ " in this figure. 
tion of fixation on a certain HFP was defined as follows: Time interval (TI) between a certain CdS photocell and the next one or the following response key was recorded as shown in Fig. 2. Duration of fixation of a particular HFP within a specific response trial (i.e., $\mathrm{HFD}_{i, j}$ ) was defined as $\mathrm{TI}_{i, j+1}$

$$
\mathrm{HFD}_{i, f}=\mathrm{TI}_{i, j+1} \text {. }
$$

Reaction time $(R T)$. Time interval between one response and the next one was defined as RT, calculated from the sequential data found in Fig. 2 as follows:

$$
\mathrm{RT}_{i}=\mathrm{TI}_{i, 0}+\sum_{j=1}^{n} \mathrm{TI}_{i, j} .
$$

For example, the second reaction time of Fig. $2\left(\mathrm{RT}_{2}\right)$ was calculated as follows:

$$
\mathrm{RT}_{2}=2.6+(.76+1.12+.51) .
$$

Duration of stimulus exposition (SED). The real duration when a stimulus was exposed on the screen was defined as SED. When a subject pushed a response key, it took about $1.18 \mathrm{~s}$ to present the next slide on the screen. So the SED on $i$-th trial was defined as

$$
\mathrm{SED}_{i}=\mathrm{RT}_{i}-1.18
$$

Strategies of head movements. 1. Convergent strategy and divergent strategy. If a response key was pushed while the HFP was in the same location as that of response key, this was defined as convergent strategy (C-st). If the last HFP on that trial was not equal to the response key, it was defined as divergent strategy (D-st).

2. Searching strategy and anticipative strategy. If a HFP was the same as the location of the target at the moment of presentation of the stimulus, it was defined as an anticipative strategy (A-st) on that trial. If the subject required some searching activities to find the target, it was categorized as a searching strategy (S-st).
Table 1

Results of ANOVA on reaction time

\begin{tabular}{lcccc}
\hline Factor & SS & $d f$ & MS & $F$ \\
\hline $\begin{array}{c}\text { Established } \\
\text { hypothesis } \\
\text { (Block) }\end{array}$ & 1.546 & 4 & .387 & $10.6489 * * *$ \\
$\begin{array}{c}\text { Individual } \\
\text { differences }\end{array}$ & .403 & 6 & .067 & 1.8187 \\
Error & .886 & 24 & .037 & \\
\hline Total & 2.835 & 34 & &
\end{tabular}

Results

\section{Reaction Time Before and After Hypothesis Formation}

The mean RT during hypothesis formation (MRHF) was computed for each subject. To calculate the mean $\mathrm{RT}$ on hypothesis-confirmation trials (MRHC), trials after hypothesis formation were divided into four blocks, block-size being equal to the number of trials required for each subject to form hypothesis, and the mean RT in each of four blocks $\left(\mathrm{MRHC}_{n} ; n=1-4\right)$ was computed; the trials after the four blocks were discarded from the computation. The first trial after hypothesis formation was also neglected because the RT included not only HFD but also time loss generated by the pushing of the key to report hypothesis formation. These MRHF and MRHCs were regarded as the true value of reaction time before and after hypothesis formation.

A two-way ANOVA was carried out to confirm whether individual differences were significant or not. As is evident in Table 1 , the main effect of individual differences was not significant. It was ascertained, however, that the effect of the established hypothesis was significant $(F=10.6489, d f=4 / 24, p<.001)$. Then a $t$-test of the difference between MRHF and each MRHC was carried out. As was expected, MRHF was greater than any of MRHCs $\left(p<.001\right.$ on $\mathrm{MRHC}_{1}$, $\mathrm{MRHC}_{2}$ and $\mathrm{MRHC}_{4}, \quad p<.01$ on 
$\mathrm{MRHC}_{8}$ ).

\section{Head Movements}

Head movements during hypothesis formation (HMHF) and those during hypothesis confirmation $\left(\mathrm{HMHC}_{n}\right)$ were compared.

Convergent strategy and divergent strategy. Head movements on the trials in which head movements existed were categorized according to the category of C-st and D-st. In order to assess the effect of the blocks ( $\mathrm{HMHF}, \mathrm{HMHC}_{1}$ to $\mathrm{HMHC}_{4}$ ), all trials in the blocks were analyzed. The $\chi^{2}$ value was $1.5018(d f=4)$. There were no significant block effects.

Searching strategy and anticipative strategy. Convergent strategies were further analyzed in terms of the S-st and A-st. The percentages of these two strategies varied with the block. Figure 3 shows the results.

The $\chi^{2}$-test showed that the percentages of S-st and A-st varied significantly $\left(\chi^{2}=9.5616, d f=4, p<.05\right)$ with the block. The percentages of S-st and A-st on each HMHC block was compared with the HMHF block. On the first HMHC block $\left(\mathrm{HMHC}_{1}\right)$, significant $\chi^{2}$ value could not be obtained. On $\mathrm{HMHC}_{2}, \mathrm{HMHC}_{3}$ and $\mathrm{HMHC}_{4}$, the percentages of S-st to A-st

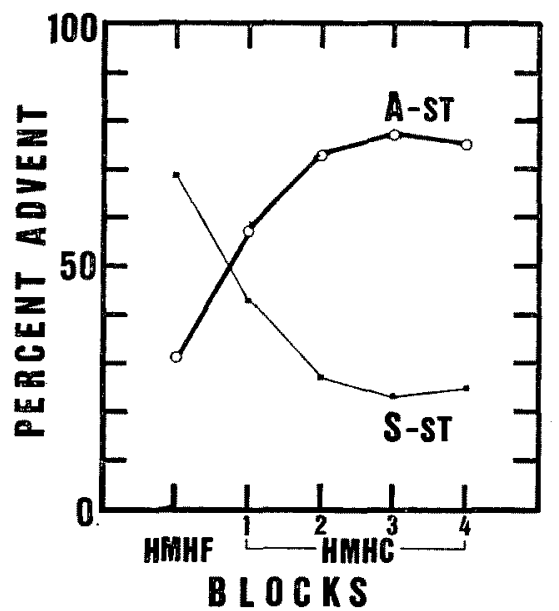

Fig. 3. Percentages of anticipative strategy (Ast) and searching strategy (S-st). was significantly reversed $(p<.05)$, i.e., S-st appeared more in the HMHF block, while A-st appeared more in $\mathrm{HMHC}_{n}$ $(n=2$ to 4 ) blocks.

\section{Discussion}

\section{A Tentative Model}

Activities related with the hypothesis could be illustrated as follows.

Subjects may scan the screen in search of the target under the set of " searching the target". When they find it, they push the response key to report its discovery. If not, they go on searching. Some trials later, a certain hypothesis may be formed concerning the spatial location of the target.

After that kind of pattern hypothesis is established, they may adopt the strategy of waiting for the target to appear in advance of the presentation of the stimulus, where they process the information of the stimulus instantly and report that they found the target. If the target does not appear in the place they expected, they will return to the earlier phase of establishing the hypothesis.

\section{$A$ Test of the Model}

The results obtained here supported the quantitative aspect of the model, i.e., it required much time until a hypothesis was established. After the establishment of the hypothesis, however, the time could be reduced.

The long RT on trials before hypothesis formation reflects the inefficient strategy, searching strategy (S-st), while the relatively short RT on trials after hypothesis formation reflects anticipative strategy (A-st). The results suggest the following: If it were not for hypothesis, an individual would scan the problem space in search of the target he has to find. He may accidentally find the target. With a hypothesis, however, he can adopt the strategy of anticipating for the target. The qualitative analysis stated above also 
supported the model.

Head Movements as Indices of InformationSearching Activities

Head movements are more easily detectable than eye movements. Although the problem of the interchangeability of the eye movements and the head movements under the constraints of the visual field still remains in question, head movements could be regarded as one of the most available measures of human information-searching activities.

\section{References}

Bruner, J. S. 1951 Personality dynamics and the process of perceiving. In R. R. Blake \& G. V. Ramsey (Eds.), Perception: An approach to person- ality. New York: Ronald Press Company. Pp. 121-147.

Bruner, J. S., \& Postman, L. 1949 Perception, cognition, and behavior. Journal of Personality, $18,14-31$.

Gregory, R. L. 1974 Choosing a paradigm for perception. In E. C. Craterrette \& M.P. Friedman (Eds.), Handbook of perception: Historical and philosophical roots of perception. Vol. 1. New York: Academic Press. Pp. 255-283.

Postman, L. 1951 Toward a general theory of cognition. In J. H. Rohrer \& M. Scherif (Eds.), Social psychology at the crossroads. New York: Harper \& Brothers. Pp. 242-272.

Power, R.P. 1978 Hypothesis in perception: Their development about unambiguous stimuli in the environment. Perception, 7, 105-111.

Salthouse, T. A., \& Danziger, W. L. 1978 Perception as hypothesis testing. Bulletin of the Psychonomic Society, 11, 197-199.

(Received June 27, 1983; accepted March 10, 1984) 